\title{
Impact of overgrazing and documentation of wild fodder plants used by Gujjar and Bakerwal Tribes of district Rajouri (J\&K), India
}

\author{
Tajinder Singh*, Amandeep Singh and L.R. Dangwal \\ Garhwal-249199 (Uttarakhand), INDIA \\ *Corresponding author. E-mail: tajkhalsa@gmail.com \\ Received: June 11, 2014; Revised received: February 24, 2016; Accepted: May 16, 2016
}

Herbarium and Plant Systematic Lab., H.N.B Garhwal Central University, SRT Campus, Badshahithaul Tehri

\begin{abstract}
The present study aims to documents the wild plants used as fodder by Gujjar and Bakarwal tribes and their impact of utilization and overgrazing in the forests of district Rajouri (J\&K), India. During the study period two blocks which were densely inhabited by Gujjar and Bakkerwal tribes were selected (Nowshera and Budhal) and frequent field trips were also made on other blocks of the district where the tribal people may reached. A total of 63 plants species were reported from the study area belong to 33 families and 51 genera, of all these 63 species, 22 were trees, 11 were shrubs and 30 were herbs. During the study it was observed that, due overgrazing, lopping, and cutting of forest plants in large scale by the tribes and local inhabitants for the expansion of agricultural lands and local settlements outside and inside of the forests and the number of livestock reported much more than the carrying capacity of forest, which resulted into the threatening of some plant species. It is also observed that some species like Pinus wallichiana and Cedrus deodara were not be reported in the area due to their commercial utilization and overgrazing purposes, and also due to their more litter fall which is not good for the growth of ground flora. The tribes sometimes also employ forest fire for better fodder, which leads to great loss to the regeneration of forest plants.
\end{abstract}

Keywords: District Rajouri, Fodder Plants, Forest, Gujjar and Bakarwal Tribes, Nomadic life, Overgrazing

\section{INTRODUCTION}

District Rajouri is one of the important district of $\mathrm{J} \& \mathrm{~K}$ having rich plant diversity. The district lies in western circle of Jammu division and bounded by district Poonch in North, district Reasi in West and Pakistan in South and East. It lies in between in $30^{\circ}-50^{\circ} \mathrm{N}$ to $33^{\circ}$ $30^{\circ} \mathrm{N}$ longitude and $74^{\circ} \mathrm{E}$ to $74^{\circ}-10^{\prime} \mathrm{E}$ latitude with altitudinal range from 370-4600 masl. Gujjar and Bakarwal tribes are constitutes the major segment of the district population and the tribal race of $\mathrm{J} \& \mathrm{~K}$ state, leading the nomadic life (Schultes, 1962; Prashad, 1992; Kapoor et al., 1994; Gaur, 2008; Rashid et al., 2008), who graze their herds of sheep, goat and cattle from south of Pir Panjal range to alpine pasture of the greater Himalaya in north, using mainly forest resources to fulfill their needs like food, fodder, forage, shelter, fuel wood, fibre, medicines, etc. As their nomadic life, some Gujjar tribes have their own permanent houses but they also migrate to alpine zone during spring as well as summer season. The Bakarwal tribes are purely nomadic, they migrate from one place to another place for obtaining better grazing opportunities (Prashad, 1992; Kapoor et al., 1994), they are known for rearing goats and some other animals such as sheep, horses etc. The Gujjar tribe mainly rears cattle but sometimes all animals. In winter season they migrates from alpine zone to subtropical zone of the district Kalakote, Nowshera, Sunderbani, and in summer season they migrate alpine zone of Pir Panjal, although sometimes they migrate to Kashmir for grazing and business purposes by the sale of their animals. The primary occupation of these tribes is livestock and secondary forest product on which they depend. Forest played a significant role in the development of civilization; they are not only indispensable sources of food, fuel, fodder, and material for shelters but also are essential for the protection and maintenance of natural environment (Negi et al., 1997).

Fodder plants used as a crude food for livestock, usually the food is composed of entire plants including leaves, stalks and grains etc. Generally straw of various crops and wild grasses as well as plant residues of pulses constitute the major fodder components. Cattle generally graze the grasses that constitute the major element of fodder. Cattle and other animals also browse green leaves and twigs of many other shrubs and trees in the hilly forest, of these some plants are dried and stored for drought condition by the tribes for the fodder of cattle. Tree and shrubs can supplement the quality and quantity of pastures for grazing live stock. They are an effective insurance against seasonal feed shortage or the risk of drought (Schultes, 1962; Prashad, 1992; 
disturbances and livestock grazing also cause change in species number, tree density and basal area (Rao et al., 1990). Unrestricted and open accessibility may cause enhance utilization of forest resource and this may eventually leads to species poor state (Vetaas, 1993; Murali et al., 1996).

Besides exploring floristic diversity and invention of the plant resources of the western Himalayas and the state $\mathrm{J} \& \mathrm{~K}$, documentation of the traditional knowledge on the ethno-botanical utilization of plants has been workout by several workers during last two decades (Kachroo and Nahvi, 1976; Gupta et al., 1982; Kaul et al., 1987; Jain, 1991; Lal et al., 1996; Singh and Kumar, 2000; Anjula et al., 2007; Dangwal et al., 2011, 2012a, 2012b, 2013, 2014).The fodder plants can be utilized for agro-forestry purposes in the adverse condition of drought and also take some steps for the conservation of these plants and cultural heritage of the tribes. The present study will be helpful for obtaining the present scenario of forest. It would also provide information regarding the range of impact on the forest plants. The out coming of this study will also utilize for conservation purposes. Study will also be helpful for the development of social and agro forestry plants in the area which ultimately releases the grazing pressure from the forest. Thus, the main focus of the present study is to document the some wild fodder plants of the district Rajouri used by Gujjar and Bakarwal tribes and their impact on forest plants.

\section{MATERIALS AND METHODS}

Frequent field trips were made during the period of 2010-2011 to survey the inhabiting area of the Gujjar and Bakarwal tribes. Two blocks (Nowshera and Budhal) of the district Rajouri were selected based on seasonal migration of Gujjar and Bakarwal tribes. Monthly field trips were made in both blocks of the district for the collection and more information of fodder plants. Interviews were conducted from the local inhabitants regarding the collection of fodder plants, parts used as fodder and their available vernacular names. The collected plants were identified with the help of published floras, literature and monographs, Hooker (1906); Sharma and Kachroo (1983); Swami and Gupta (1998); Gaur (1999). The authentication of the collected plants was confirmed from the regional Herbaria at Botanical Survey of India, Northern Circle (BSD), Dehradun, Herbarium of Forest Research Institute (DD), Dehradun and HNB Garhwal University, Herbarium (GUH), Srinagar Garhwal, Uttarakhand. These plants were deposited in the Herbarium of Department of Botany, S.R.T. Campus Badshahi Thaul, district Tehri Garhwal.

\section{RESULTS AND DISCUSSION}

Main aim of the present study is to document the forest fodder plants commonly used by the Gujjar and Bakarwal tribes in drought conditions (scarcity of fodder). In the present study a total of 63 fodder plants were reported from the study area. The reported plants belonging to 32 families and 51 genera, of these 22 were trees, 11 were shrubs and 30 were herbs. Family wise listing of fodder plants have been arranged with their botanical name, local name, flowering and fruiting season and parts used as fodder as given in Table 1. In contrast to present study Dangwal and Amandeep (2012) studied the fodder weeds of district Rajouri and reported 57 species belonging to 22 families, whereas Rashid and Anil (2012) studied the fodder plants of same district and reported 68 fodder plant species. Singh et al., (2014) was reported 65 grassy weed species belonging to 05 families, while Dangwal and Tajinder, (2013) reported 29 species used as medicine by Gujjar tribe belonging to 22 families of district Rajouri. Based on the consumption as fodder, herbs are the dominant as compared to shrubs and trees shown in Fig.1. In herbs, the family Poacae and Fabacae have shown dominancy and mostly browsed and grazed by cattle and other animals of the tribes.

The average numbers of livestock per family commonly known as Kumba have been shown in Table 2. The yearly increasing population of livestock and these tribes mainly depend on forest for their livestock. Study also revealed that the number of livestock is much more than the carrying capacity of forest. In this area due overgrazing, lopping, and cutting of forest plants in large scale by the tribes and local inhabitants for the expansion of agricultural lands and local settlements outside and inside of the forests, which resulted into the threatening of some plant species. These plants are Quercus leucotrichophora, Q. semecarpifolia, Spiraea spp. etc. Similar to our present study, Kumar and Shahabuddin (2005) studied the impact of uncontrolled common land grazing and overstocking of live stock on forest. Ives (2006) studied the negative impact of land use in the Indian Himalayas. Negi and Todaria (1993) also observed the impact of high concentration of grazing animals in the growing regions which also harm the forest to the large extent.

The vegetation of Oak forest decreasing every year

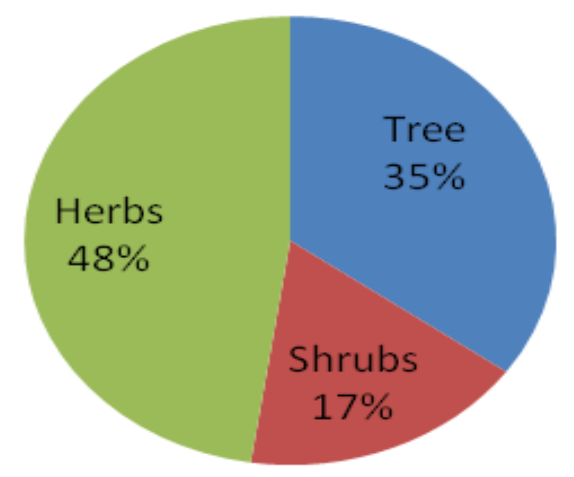

Fig. 1. Diversity of trees, shrubs and herbs used as fodder 


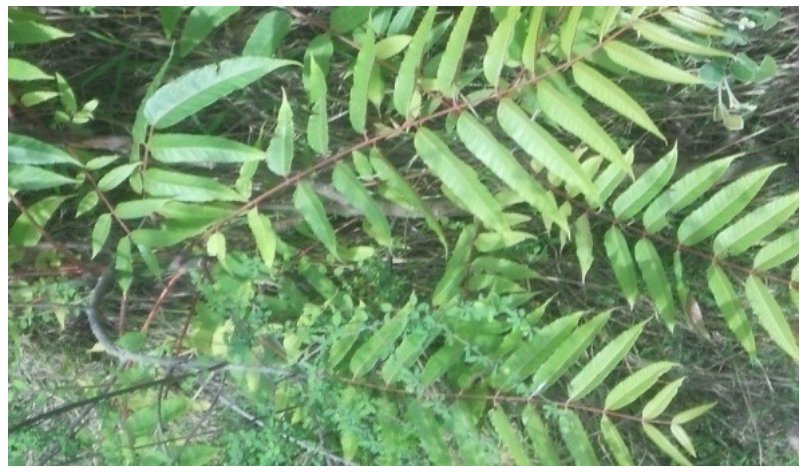

Toona serrata
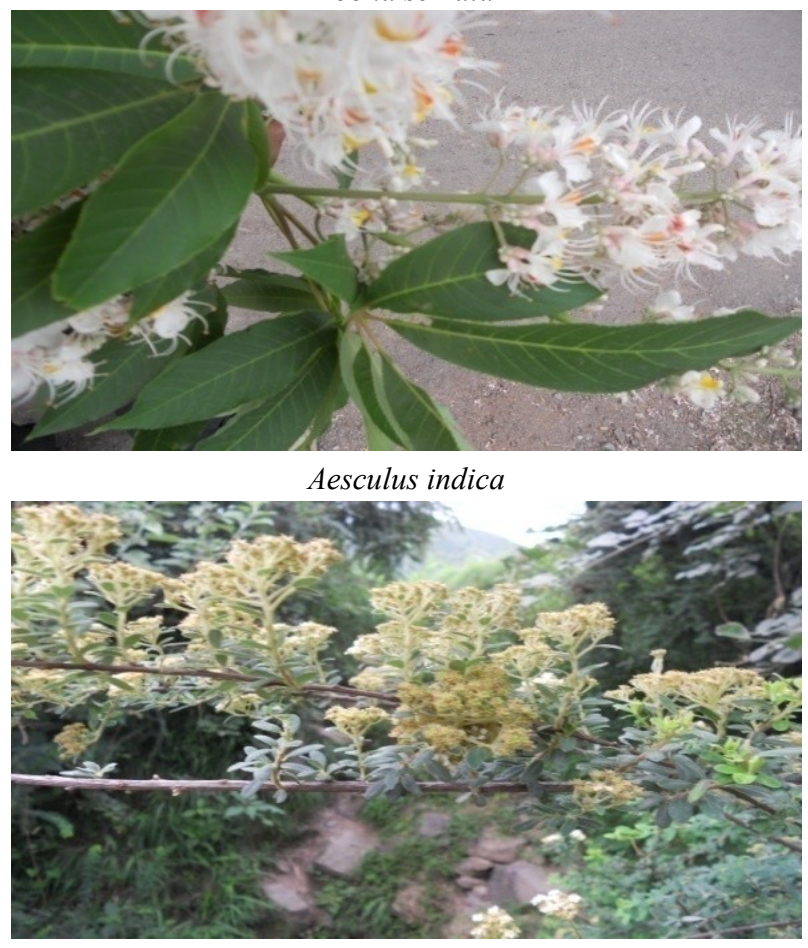

Spiraea canescens

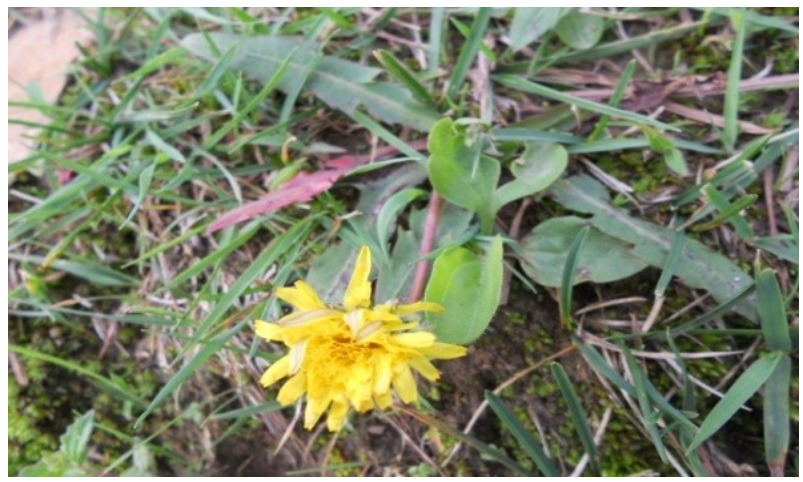

Taraxacum officinale

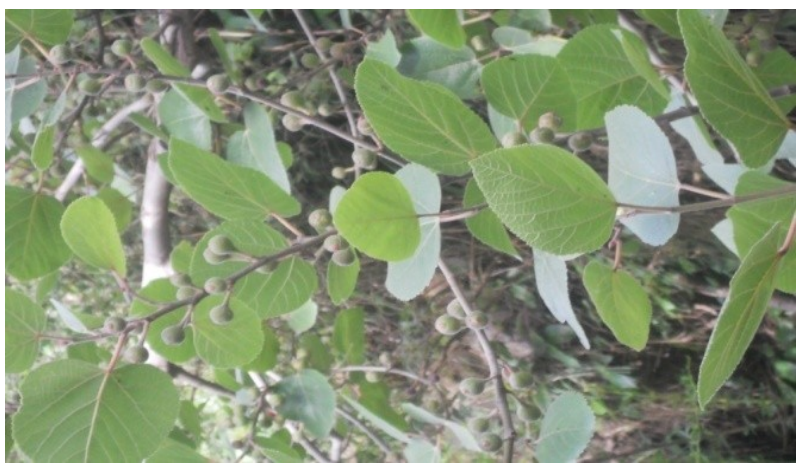

Ficus palmata

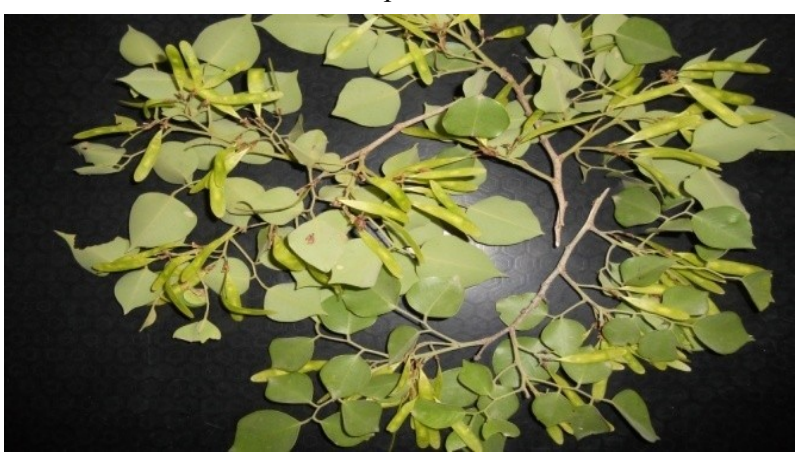

Dalbergia sissoo

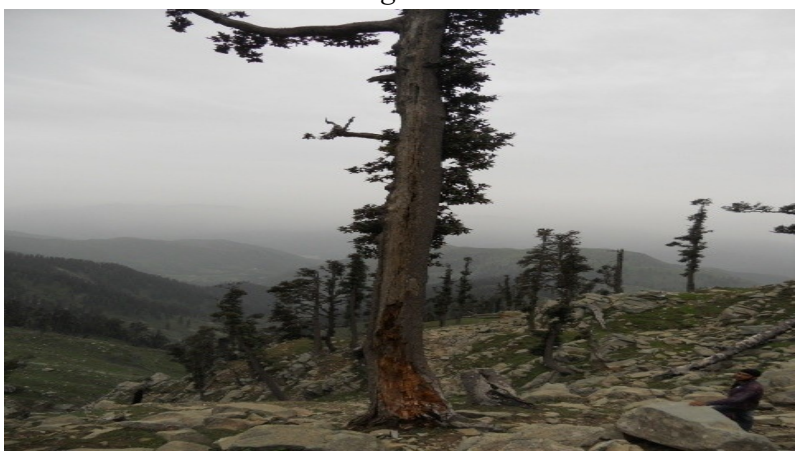

Quercus semecarpifolia

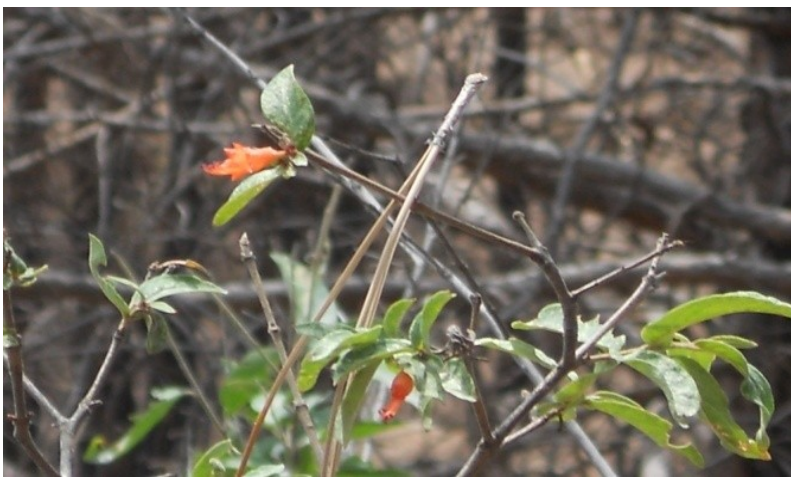

Woodfordia fruticosa

Plate 1. Plants used as fodder.

as shown in Plates 2 and 3, not only for fodder purpose but also cutting of trees for their multipurpose such trees are Pinus roxburghii, Taxus baccata, Abies pindrow, Picea smithiana etc. It is also observed that some species like Pinus wallichiana and Cedrus deodara could not be found in the area due to their commercial utilization and overgrazing purposes, and also due to their more litter fall which is not good for the growth of ground flora. If litter fall is more, the tribes sometimes also employ forest fire for better fodder which leads to the great loss to the regeneration of forest plants. 
Table 1. Documentation of forest fodder plants used by Gujjar and Bakarwal Tribes.

\begin{tabular}{|c|c|c|c|c|c|c|}
\hline $\begin{array}{l}\text { S. } \\
\text { N. }\end{array}$ & Family & Botanical Name & Habit & $\begin{array}{l}\text { Local } \\
\text { name }\end{array}$ & $\begin{array}{l}\text { Flowering and fruiting } \\
\text { season }\end{array}$ & $\begin{array}{l}\text { Plant parts used as } \\
\text { fodder }\end{array}$ \\
\hline $\mathrm{T}$ & Acanthaceae & $\begin{array}{l}\text { Dicliptera bupleuroides Nees } \\
\text { in Wallich. }\end{array}$ & Herb & - & $\begin{array}{l}\text { F1.: Sept.-Nov. } \\
\text { Fr.: Oct.-Dec. }\end{array}$ & $\begin{array}{l}\text { The whole plant used as } \\
\text { fodder. }\end{array}$ \\
\hline 2 & Anacardiaceae & $\begin{array}{l}\text { Lannea coromandelica } \\
\text { (Houttun) Mereill }\end{array}$ & Tree & Kalma & $\begin{array}{l}\text { Fl.: Mar.-Apr. } \\
\text { Fr.: Jun.-Aug. }\end{array}$ & Leaves used as fodder. \\
\hline 3 & Apocynaceae & $\begin{array}{l}\text { Carissa opaca Stapf ex } \\
\text { Haines }\end{array}$ & Shrub & Granda & $\begin{array}{l}\text { Fl.: Jan.-Mar. } \\
\text { Fr.: Apr.-Jun. }\end{array}$ & $\begin{array}{l}\text { Leaves browsed by } \\
\text { sheep and goats. }\end{array}$ \\
\hline 4 & Aquifoliaceae & $\begin{array}{l}\text { Ilex dipyrena Wallich in } \\
\text { Roxb. }\end{array}$ & Tree & Dratha & $\begin{array}{l}\text { Fl.: Mar.-Apr. } \\
\text { Fr.: Jun.-Oct. }\end{array}$ & $\begin{array}{l}\text { Leaves rarely used as } \\
\text { fodder. }\end{array}$ \\
\hline 5 & Asclepiadaceae & $\begin{array}{l}\text { Cryptolepis buchananii Roe- } \\
\text { mer \& Schultes }\end{array}$ & Herb & Dodi bale & $\begin{array}{l}\text { Fl.: Mar.-Jul. } \\
\text { Fr.: Oct.-Nov. }\end{array}$ & $\begin{array}{l}\text { Sometime browsed by } \\
\text { goats. }\end{array}$ \\
\hline 6 & Asteraceae & Bidens pilosa $\mathrm{L}$. & Herb & Saryla & Fl. \& Fr.: Mar.-Aug. & $\begin{array}{l}\text { Plant browsed as fod- } \\
\text { der. }\end{array}$ \\
\hline & & Taraxacum officinale Weber & Herb & Hand & Fl.\& Fr.: Mar.-Oct. & Used as fodder. \\
\hline 7 & Brassicaceae & Lepidium sativum L. & Herb & - & $\begin{array}{l}\text { Fl.: Feb.-Apr. } \\
\text { Fr.: Apr-May }\end{array}$ & $\begin{array}{l}\text { Browsed by } \\
\text { cattle and goats. }\end{array}$ \\
\hline 10 & Dioscoreaceae. & Dioscorea bulbifera $\mathrm{L}$. & Herb & - & $\begin{array}{l}\text { Fl.: Jul.-Nov. } \\
\text { Fr.: Oct.-Jan. }\end{array}$ & $\begin{array}{l}\text { Leaves provide good } \\
\text { fodder. }\end{array}$ \\
\hline 11 & Elaeagnaceae & $\begin{array}{l}\text { Elaeagnus parvifolia wallich } \\
\text { ex Royle. }\end{array}$ & Shrub & - & $\begin{array}{l}\text { Fl.: Mar-Apr. } \\
\text { Fr.: Jun.-Sept. }\end{array}$ & $\begin{array}{l}\text { Leaves lopped for } \\
\text { fodder. }\end{array}$ \\
\hline \multirow[t]{5}{*}{12} & Fabaceae & $\begin{array}{l}\text { Dalbergia sissoo Roxb } \\
\text { Desmodium microphyllum } \\
\text { (Thunb.) DC. }\end{array}$ & $\begin{array}{l}\text { Tree } \\
\text { Herb }\end{array}$ & $\begin{array}{l}\text { Tal } \\
-\end{array}$ & $\begin{array}{l}\text { Fl. \& Fr.: Mar.-Jun. } \\
\text { Fl. \& Fr.: Jan.-Dec. }\end{array}$ & $\begin{array}{l}\text { Leaves used as fodder. } \\
\text { Fodder for cattle. }\end{array}$ \\
\hline & & $\begin{array}{l}\text { Indigofera heterantha Wal- } \\
\text { lich ex Brandis }\end{array}$ & Shrub & - & $\begin{array}{l}\text { Fl.: May-Aug. } \\
\text { Fr.: Sept.-Nov. }\end{array}$ & $\begin{array}{l}\text { Leaves lopped for } \\
\text { fodder. }\end{array}$ \\
\hline & & Lotus corniculata L. & Herb & Nethi & $\begin{array}{l}\text { Fl.\& Fr.: Almost } \\
\text { throughout the year }\end{array}$ & Grazed by cattle. \\
\hline & & Medicago lupulina $\mathrm{L}$. & Herb & - & Fl. \& Fr.: Nov.-May & Plant used as fodder. \\
\hline & & Trifolium pratense $\mathrm{L}$. & Herb & - & Fl. \&Fr. : Jul.-Sept. & $\begin{array}{l}\text { Whole plant used as } \\
\text { fodder for cattle. }\end{array}$ \\
\hline \multirow[t]{3}{*}{13} & Fagaceae & $\begin{array}{l}\text { Vigna vexillata } \mathrm{L} \text {. } \\
\text { Quercus glauca Thunb. }\end{array}$ & $\begin{array}{l}\text { Herb } \\
\text { Tree }\end{array}$ & $\begin{array}{l}\text { Jangli Dal } \\
\text { Burai }\end{array}$ & $\begin{array}{l}\text { Fl.\& Fr.: Aug.-Nov. } \\
\text { Fl.: May-Jun. } \\
\text { Fr.: Jun.-Aug. }\end{array}$ & $\begin{array}{l}\text { Plant used as fodder. } \\
\text { Leaves used as fodder. }\end{array}$ \\
\hline & & $\begin{array}{l}\text { Quercus leucotrichophora A. } \\
\text { Camus in Riviera Sci. }\end{array}$ & Tree & Rein & $\begin{array}{l}\text { Fl.: Mar.-Aor. } \\
\text { Fr.: Oct.-Jan. }\end{array}$ & Leaves used as fodder. \\
\hline & & $\begin{array}{l}\text { Quercus semecarpifolia J.E. } \\
\text { Smith in Rees }\end{array}$ & Tree & Peli Rein & $\begin{array}{l}\text { Fl.: Mar.-Apr. } \\
\text { Fr.: Aug.-Sept. }\end{array}$ & Leaves used as fodder. \\
\hline 14 & Flacourtiaceae & Flacourtia indica (Burm.f.) & Tree & Jela & $\begin{array}{l}\text { Fl.: Feb.-Mar. } \\
\text { Fr.: Apr.-Jun. }\end{array}$ & Leaves used as fodder. \\
\hline 15 & Hippocastanaceae & $\begin{array}{l}\text { Aesculus indica (Coler. ex } \\
\text { Cambess) Hook. in Curtis }\end{array}$ & Tree & Bankhori & $\begin{array}{l}\text { Fl.: Mar.-Apr. } \\
\text { Fr.: Jul.-Aug. }\end{array}$ & Leaves used as fodder. \\
\hline \multirow[t]{2}{*}{16} & Hypericaceae & $\begin{array}{l}\text { Hypericum oblongifolium } \\
\text { Choisy. }\end{array}$ & Shrub & - & $\begin{array}{l}\text { Fl.: Mar.-Apr. } \\
\text { Fr.: May-Jun. }\end{array}$ & $\begin{array}{l}\text { Leaves looped for } \\
\text { fodder. }\end{array}$ \\
\hline & & Hypericum perforatum $\mathrm{L}$. & Herb & - & $\begin{array}{l}\text { Fl. \& Fr.: } \\
\text { Throughout the year }\end{array}$ & Leaves used as fodder \\
\hline 17 & Lythraceae & Woodfordia fruticosa (L.) & Shrub & - & $\begin{array}{l}\text { Fl.: Jan.-Apr. } \\
\text { Fr.: Apr.-Jun. }\end{array}$ & $\begin{array}{l}\text { Leaves used as } \\
\text { fodder for goats. }\end{array}$ \\
\hline \multirow[t]{2}{*}{18} & Meliaceae & Melia azedarach L. & Tree & Dreek & $\begin{array}{l}\text { Fl.: Mar.-Apr. } \\
\text { Fr.: Apr.-May }\end{array}$ & Leaves used as fodder. \\
\hline & & $\begin{array}{l}\text { Toona serrata (Royle) M. } \\
\text { Roemer. }\end{array}$ & Tree & Toon & $\begin{array}{l}\text { Fl.: May-Jun. } \\
\text { Fr.: Sept.-Dec. }\end{array}$ & Leaves used as fodder. \\
\hline 19 & Mimosaceae & Acacia eburnia (L.f.) Willd. & Shrub & Patki & $\begin{array}{l}\text { Fl.: Sept.-Nov. } \\
\text { Fr.: Feb.-Apr. }\end{array}$ & Foliage used as fodder. \\
\hline & & $\begin{array}{l}\text { Albizia lebbeck (L.) Benth. } \\
\text { in Hook. }\end{array}$ & Tree & Sree & $\begin{array}{l}\text { Fl.: Apr.-Jun. } \\
\text { Fr.: Sept.-Dec. }\end{array}$ & $\begin{array}{l}\text { Leaves provide palat- } \\
\text { able fodder for cattle. }\end{array}$ \\
\hline & & $\begin{array}{l}\text { Acacia modesta } \\
\text { Wallich. }\end{array}$ & Tree & Playi & $\begin{array}{l}\text { Fl.: Mar.-May } \\
\text { Fr.: Oct.-Dec. }\end{array}$ & $\begin{array}{l}\text { Foliage as fodder of } \\
\text { goats and sheep. }\end{array}$ \\
\hline \multirow[t]{3}{*}{20} & Moraceae & Ficus auriculata Lour & Tree & Tose & $\begin{array}{l}\text { Fl.: Mar.-May. } \\
\text { Fr.: Jun.-Jul. }\end{array}$ & $\begin{array}{l}\text { Leaves are the good } \\
\text { fodder for cattle. }\end{array}$ \\
\hline & & Ficus palmata Forsk. & Tree & Fagwara & $\begin{array}{l}\text { Fl.: May-Jun. } \\
\text { Fr.: Jun.-Aug. }\end{array}$ & $\begin{array}{l}\text { Leaves and twigs good } \\
\text { fodder. }\end{array}$ \\
\hline & & $\begin{array}{l}\text { Ficus semicordata Buch.- } \\
\text { Ham.ex J. E. Smith in Rees. }\end{array}$ & Tree & - & $\begin{array}{l}\text { Fl.: May- Jun. } \\
\text { Fr.: Jun.-Oct. }\end{array}$ & $\begin{array}{l}\text { Leaves and twigs } \\
\text { lopped for fodder. }\end{array}$ \\
\hline
\end{tabular}




\begin{tabular}{|c|c|c|c|c|c|c|}
\hline 21 & Oleaceae & Olea ferruginea Royle. & Tree & Kaaw & $\begin{array}{l}\text { Fl.: Feb.-Apr. } \\
\text { Fr.: Oct.-Nov. }\end{array}$ & Leaves used as fodder. \\
\hline 22 & Oxalidaceae & Oxalis corniculata L. & Herb & Kathimli & $\begin{array}{l}\text { Fl. \& Fr.: Almost } \\
\text { throughout the year. }\end{array}$ & Grazed by cattle. \\
\hline \multirow[t]{10}{*}{23} & \multirow[t]{10}{*}{ Poaceae } & Alloteropsis cimicina (L.) & Herb & - & Fl. \& Fr.: Aug.-Nov. & $\begin{array}{l}\text { Whole plant used as fod- } \\
\text { der. }\end{array}$ \\
\hline & & $\begin{array}{l}\text { Capillipedium assimile } \\
\text { (Steudel) A. Camus in Le- } \\
\text { comte. }\end{array}$ & Herb & Gass & Fl. \& Fr.: Aug.-Dec. & Plant is a good fodder. \\
\hline & & Cenchrus ciliaris L. & Herb & Seta Gass & Fl. \& Fr. Aug.-Sept. & $\begin{array}{l}\text { Plant used as fodder for } \\
\text { cattle. }\end{array}$ \\
\hline & & $\begin{array}{l}\text { Cynodon dactylon (L.) Per- } \\
\text { soon. }\end{array}$ & Herb & Khabal & Fl.\&Fr.: Jan.-Dec. & Grazed by Cattle. \\
\hline & & $\begin{array}{l}\text { Echinochloa colona (L.) } \\
\text { Link. }\end{array}$ & Herb & Gass & Fl. \& Fr.: Jul.-Oct. & Leaves used as fodder. \\
\hline & & Panicum antidotale Retz. & Herb & Gass & Fl.\&Fr.: Dec.-Jan. & $\begin{array}{l}\text { Whole plant used as fod- } \\
\text { der. }\end{array}$ \\
\hline & & $\begin{array}{l}\text { Paspalidium flavidum } \\
\text { (Michaux) Scribn. }\end{array}$ & Herb & Gass & Fl. \& Fr.: Jul.-Nov. & Used as fodder. \\
\hline & & Setaria glauca (L.) P.Beav. & Herb & Seta gass & Fl. \& Fr.: Aug.-Nov. & $\begin{array}{l}\text { Whole plant used as fod- } \\
\text { der for cattle. }\end{array}$ \\
\hline & & Setaria viridis (L.) P.Beauv. & Herb & Seta gass & Fl. \& Fr.: Aug.-Dec. & $\begin{array}{l}\text { Hay and green grass as } \\
\text { fodder. }\end{array}$ \\
\hline & & Themeda triandra Forsk. & Herb & gass & Fl. \& Fr.: Aug.-Oct. & Fodder for cattle. \\
\hline \multirow[t]{3}{*}{24} & \multirow[t]{3}{*}{ Polygonaceae } & Rumex hastatus D.Don. & Herb & - & $\begin{array}{l}\text { Fl.: Feb.-Jun. } \\
\text { Fr.: Jun.-Oct. }\end{array}$ & Rarely used as fodder. \\
\hline & & Rumex dentatus $\mathrm{L}$. & Herb & Arfali & Fl. \& Fr.: Feb.-May & $\begin{array}{l}\text { Also used as miner fod- } \\
\text { der. }\end{array}$ \\
\hline & & Rumex nepalensis Sprengel & Herb & Hebli & $\begin{array}{l}\text { Fl.: Apr.-Aug. } \\
\text { Fr.: Aug.-Oct. }\end{array}$ & Rarely used as fodder. \\
\hline \multirow[t]{3}{*}{25} & \multirow[t]{3}{*}{ Ranunculaceae } & Clematis grata Wallich. & $\begin{array}{l}\text { Climber } \\
\text { Herb }\end{array}$ & Tri Bale & $\begin{array}{l}\text { Fl.: Aug.-Oct. } \\
\text { Fr.: Oct.-Dec. }\end{array}$ & $\begin{array}{l}\text { Leaves used as fodder for } \\
\text { sheep and goats. }\end{array}$ \\
\hline & & $\begin{array}{l}\text { Clematis montana Buch.- } \\
\text { Ham. ex DC. }\end{array}$ & $\begin{array}{l}\text { Climber } \\
\text { herb }\end{array}$ & Tri Bale & Fl. \& Fr. : Mar.-Aug. & $\begin{array}{l}\text { Leaves browsed by sheep } \\
\text { and goats. }\end{array}$ \\
\hline & & Ranunculus hirtellus Royle & Herb & - & $\begin{array}{l}\text { Fl.: Jul.-Aug. } \\
\text { Fr.: Aug.-Sept. }\end{array}$ & Plant browed by goats. \\
\hline \multirow[t]{2}{*}{26} & \multirow[t]{2}{*}{ Rhamnaceae } & $\begin{array}{l}\text { Ziziphus glaberrima } \\
\text { (Sedgwick) }\end{array}$ & Tree & Bari & $\begin{array}{l}\text { Fl.: Sept.-Oct. } \\
\text { Fr.: Dec.-Feb. }\end{array}$ & $\begin{array}{l}\text { Browsed by sheep and } \\
\text { goats. }\end{array}$ \\
\hline & & Ziziphus mauritiana Lam. & Shrub & Bari & $\begin{array}{l}\text { Fl.: Jun.-Aug. } \\
\text { Fr.: Feb.-Mar. }\end{array}$ & $\begin{array}{l}\text { Browsed by sheep and } \\
\text { goats. }\end{array}$ \\
\hline \multirow[t]{2}{*}{27} & \multirow[t]{2}{*}{ Rosaceae } & $\begin{array}{l}\text { Pyrus pashia Buch.-Ham. ex } \\
\text { D.Don. }\end{array}$ & Tree & Kathari & $\begin{array}{l}\text { Fl.: Feb.-Mar. } \\
\text { Fr.: May-Dec. }\end{array}$ & $\begin{array}{l}\text { Leaves and twigs used as } \\
\text { fodder. }\end{array}$ \\
\hline & & Spiraea canescens D.Don & Shrub & Dhala & $\begin{array}{l}\text { Fl.: May-Jun. } \\
\text { Fr.: Jun.-Oct. }\end{array}$ & $\begin{array}{l}\text { Branches looped for } \\
\text { fodder. }\end{array}$ \\
\hline \multirow[t]{2}{*}{28} & \multirow[t]{2}{*}{ Salicaceae } & Salix acemophylla Boissier & Tree & Kankori & $\begin{array}{l}\text { Fl.: Feb.-Mar. } \\
\text { Fr.: Apr.-May }\end{array}$ & Leaves used as fodder. \\
\hline & & $\begin{array}{l}\text { Salix disperma Roxb. ex } \\
\text { D.Don }\end{array}$ & Tree & - & Fl.\& Fr.: Mar.-Jul. & Leaves used as fodder. \\
\hline 29 & Smilaceae & $\begin{array}{l}\text { Salix tetrasperma Roxb. } \\
\text { Smilax parviflora Wall }\end{array}$ & $\begin{array}{l}\text { Tree } \\
\text { Climber }\end{array}$ & $\begin{array}{l}\text { - } \\
\text { Kuler chog } \\
\text { or kalra } \\
\text { bale }\end{array}$ & $\begin{array}{l}\text { Fl.\& Fr.: Sept.-Dec. } \\
\text { Fl.: Apr.-Jun. }\end{array}$ & $\begin{array}{l}\text { Leaves used as fodder. } \\
\text { Lopped as fodder. }\end{array}$ \\
\hline 30 & Tiliaceae & $\begin{array}{l}\text { Grewia optiva J.R Drum- } \\
\text { mond ex Burret in Notizbl. }\end{array}$ & Tree & $\begin{array}{l}\text { Thaman or } \\
\text { Dhaman }\end{array}$ & $\begin{array}{l}\text { Fl.: Apr.-Jun. } \\
\text { Fr.: Aug.-Nov. }\end{array}$ & $\begin{array}{l}\text { Leaves provide good } \\
\text { fodder. }\end{array}$ \\
\hline 31 & Ulmaceae & Celtis eriocarpa Decne. & Tree & Kharik & $\begin{array}{l}\text { Fl.: Mar.-Apr. } \\
\text { Fr.: Sept.-Nov. }\end{array}$ & $\begin{array}{l}\text { Leaves provide good } \\
\text { fodder for cattle. }\end{array}$ \\
\hline 32 & Urticaceae & $\begin{array}{l}\text { Debregeasia longifolia } \\
\text { (Burm.f.)Wedd. in DC. }\end{array}$ & Shrub & - & $\begin{array}{l}\text { Fl.: Feb-Mar. } \\
\text { Fr.: Apr.-Jul. }\end{array}$ & Plant is a good fodder. \\
\hline 33 & Verbenaceae & $\begin{array}{l}\text { Premna barbata Wallich ex } \\
\text { Schauer. }\end{array}$ & Herb & - & $\begin{array}{l}\text { Fl.: Mar. - May } \\
\text { Fr.: Jun.-Aug. }\end{array}$ & Leaves used as fodder. \\
\hline
\end{tabular}

Kapoor et al., 1994). On the other hand uncontrolled common land grazing and overstocking of live stock prevent regeneration of the tree cover to some extent (Kumar and Shahabuddin, 2005) even though the negative impact of land use in the Himalayas may be overstated (Ives, 2006). Presently the forests are highly degraded due to the continuous anthropogenic disturbances and these disturbances play an important role in change as well as loss of the plant diversity of the region. These anthropogenic disturbances not only influence the soil, nutrients and water conditions but also influence climatic conditions. Human induced 

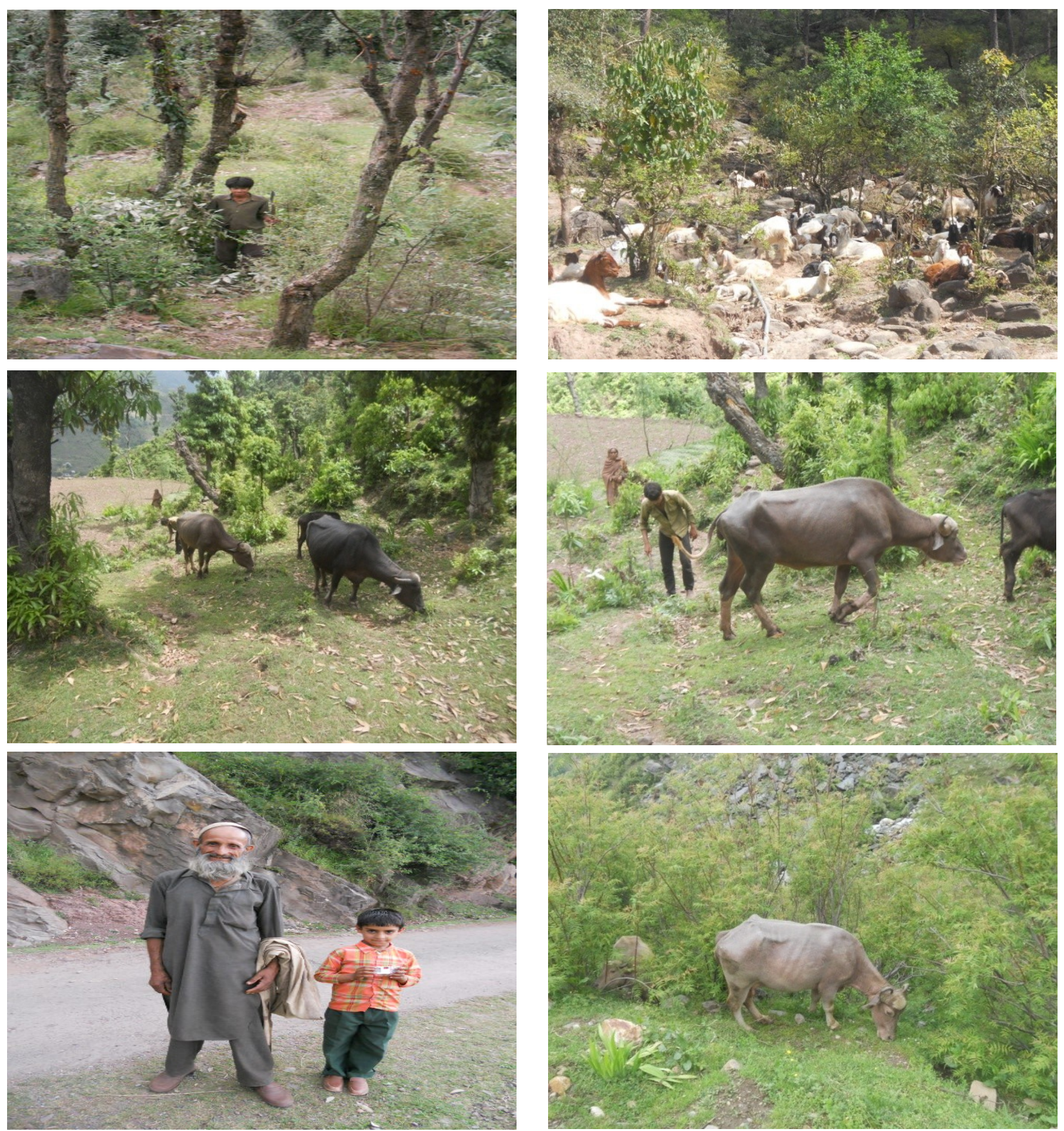

Plate 2. Gujjar and Bakarwal tribe grazing animals and using forest resources.

Table 2. Average number of livestock per family depending on the forest.

\begin{tabular}{llll}
\hline $\begin{array}{l}\text { Name of } \\
\text { livestock }\end{array}$ & Average number of livestock & & \\
\cline { 2 - 4 } & $\begin{array}{l}\text { Gujjar tribe } \\
\text { (total number per family/umba) }\end{array}$ & $\begin{array}{l}\text { Bakarwal tribe } \\
\text { (total number per family / } \\
\text { Kumba) }\end{array}$ & $\begin{array}{c}\text { Local Villagers } \\
\text { (total number per family/ } \\
\text { Kumba) }\end{array}$ \\
\hline Sheep & More than 50 & $50-100$ & $10-20$ \\
Goats & $20-100$ & $100-400$ & $20-50$ \\
Buffaloes & $7-20$ & - & $3-10$ \\
Horses & $3-7$ & $20-50$ & $2-5$ rarely \\
Other cattle & $10-15$ & - & $2-6$ \\
\hline
\end{tabular}



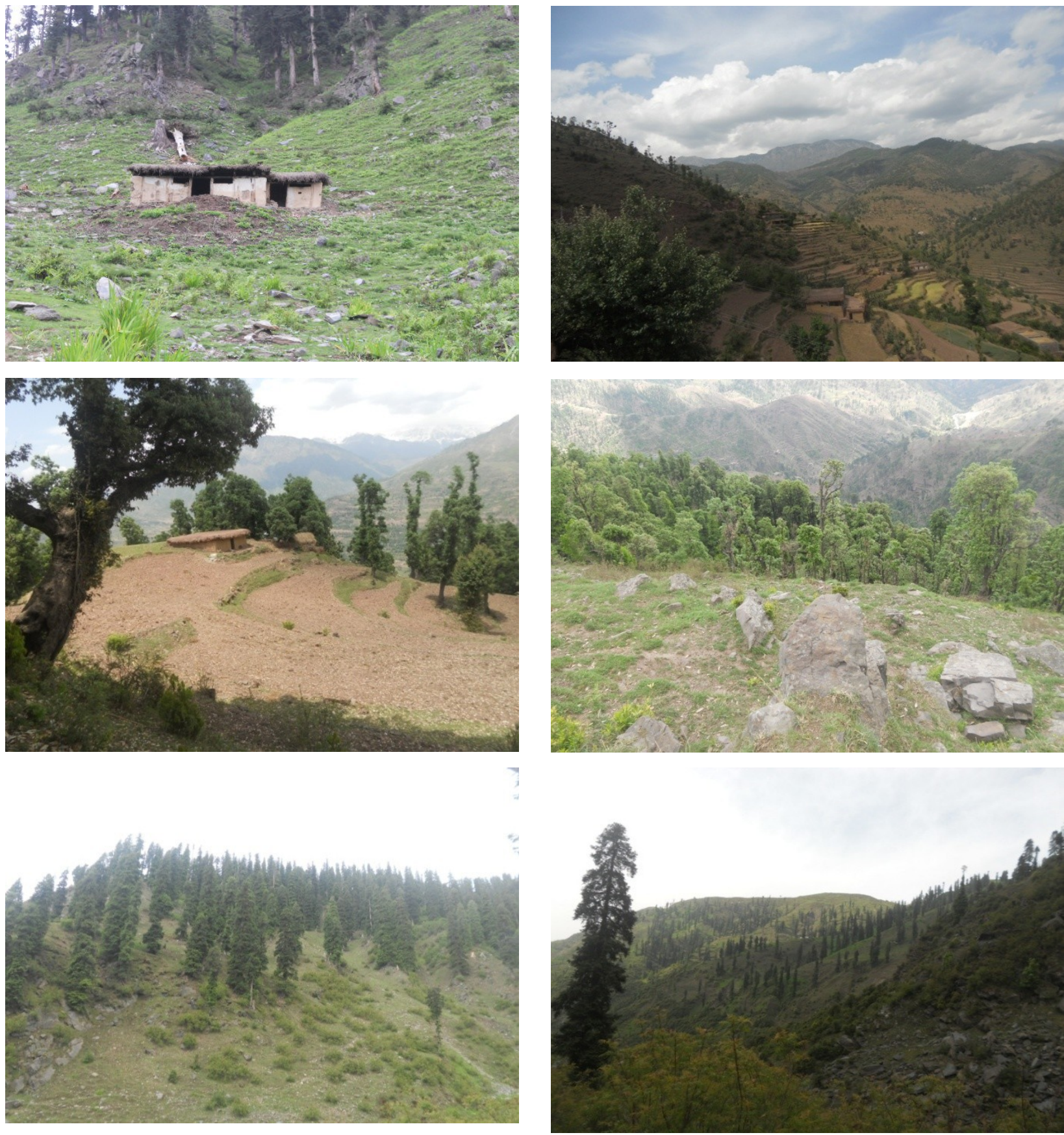

Plate 3. Showing declining, clearing of forest and expanding agricultural fields.

\section{Conclusion}

The present study reported a total of 63 plant species belong to 33 families and 51 genera, of all these 63 species, 22 were trees, 11 were shrubs and 30 were herbs. In this area due overgrazing, lopping, and cutting of forest plants in large scale by the tribes and local inhabitants for the expansion of agricultural lands and local settlements outside and inside of the forests, which resulted into the threatening of some plant species.

It is also observed that, Gujjar and Bakarwal tribes are mainly depends on forest products for their live- lihood, their economy is totally depends on livestock. Due to overgrazing as well as unfavorable environmental conditions, the forest plants and cultural heritage of these tribes is at risk. Therefore, the Govt. and Forest Officer can advised to grow agroforestry based on these forest plants as well as multipurpose plant species within the area for the conservation of forest wealth and cultural heritage of the tribes in the area.

\section{REFERENCES}

Anjula, P., Tomer, A.K., Bhandari, D.C. and Pareek S.K. (2007). Towards collection of wild relative of crop 
plants in India. J. Gen. Resour. Crop Evol.,doi. 10.007/ s10722-007-9227-4.

Dangwal, L.R., Singh, A., Sharma, A. and Singh T. (2011). Diversity of Weed species in Wheat Fields of Block Nowshera District Rajouri (J\&K), Indian J. Weed Sc. 43 (1\&2): 94-96.

Dangwal, L.R. and Singh, T. (2013). Comparative Vegetational analysis and Pinus roxburghii Sarg. regeneration in relation to their disturbances in some Chirpine forest of block Nowshera district Rajouri, J\&K, India. ISCA J. Biological Sci. 1(1): 47-54.

Dangwal, L.R. and Singh, T. (2013). Ethnobotanical study of some medicinal plants used by Gujjar tribe of district Rajouri (J\&K), India, IJAR, 3: 11-14.

Dangwal, L.R., Singh, T. and Singh, A. (2014). Exploration of wild edible plants used by Gujjar and Bakerwal tribes of District Rajouri (J\&K), India Journal of Applied and Natural Science 6 (1): 164-169

Dangwal, L.R. and Singh, A. (2012). Some common wild fodder weeds used by Gujjar tribes of district Rajouri (J\&K). ECJ, 13 (1\&2): 101-105.

Dangwal, L.R. and Singh, T. (2012a). Comparative Vegetational Analysis and Pinus roxburghii Sarg regeneration in relation to their disturbances in some Chirpine forest of Block Nowshera, district Rajouri, J\&K, India, ISCA J. Biological Sci. 1(1): 47-54.

Dangwal, L.R., Singh, T., Singh, A. and Sharma, A. (2012b). Species composition of Woody plants in Forest of Block Nowshera, District Rajouri (J\&K), India, International Journal of Current Research 4 (05) : 5-10.

Gaur, R.D. (1999). Flora of district Garhwal: North-West Himalaya (with ethnobotanical Notes). Transmedia, Srinagar Garhwal.

Gaur, R.D. (2008). Traditional dye yielding plants of Uttarakhand, India, Nat Prod Rad, 7: 154-165.

Gupta, O.P., Srivastava, T.N., Gupta, S.C. and Badola, D.P. (1982). An ethnobotany and phytochemical screening of higher altitude plants of Ladakh Part II Bull MedicoEthnobot. Res., 1:301-317.

Hooker, J.D. (1906). A sketch of the Flora of British India, Oxford Publication

Ives, J.D. (2006). Himalayan Perceptions: Environmental Change and the Wellbeing of Mountain Peoples. $2^{\text {nd }}$ edition, Kathmandu, Nepal: Himalayan Association for the Advancement of Science.

Jain, S.K. (1991). Dictionary of Indian folk medicine and ethnobotany (Deep Publication, New Delhi).

Kachroo, P. and Navi, I.M. (1976). Ethno botany of Kashmiris Forest Flora of Srinagar and plants of Neighborhood Dehra Dun, India, pp. 239-263.

Kapoor, A.K., Raha, D. and Kapoor, S. B. (1994). Ecology and man in the Himalaya. M.D. Publications, pp. 43-44. ISBN 978-81-85880-16-7.

Kaul, M.K., Sharma, P.K. and Singh, V. (1987). Ethno- botanical studies in North-West and trans-Himalaya IV. Some traditionally tea substitutes from J\&K state. $\mathrm{Hi}$ malayan Plant J., 4: 23-28.

Kumar, R. and Shahabuddin, G. (2005). Effects of biomass extraction on vegetation structum. diversity and composition of forests in Sariska Tiger Reserve, India. Environmental Conservation, 32: 248-250.

Lal, B., Vats, S.K., Singh, R.D. and Gupta, A.K. (1996). Plants used as ethnomedicine and supplement food by Gaddis of Himachal Pradesh, India, In: Jain S.K. (eds.) Ethnobiology in Human Welfare, New Delhi.

Murali, KS., Ganeshaiah, K.N., Umashankar, R. and Bawa, K.S. (1996). Extraction of non-timber forest products in the forest of Bilgiri Rangan Hill, India. 2. Impact of NTFP extraction or regeneration; population structure and species composition. Economical Botany, 50: 252-269.

Negi, A.K. and Todaria, N.P. (1993). Studies on the impact of local folk on forests of Garhwal Himalaya: Energy from Biomass. Bionzass and Bioenergy. 4(6): 445- 454.

Negi, A.K., Bhatt, B.P. and Todaria, N.P. (1997). Local population impacts on forest of Garhwal Himalaya, India. The Environmentalist, 19: 293-303,

Prashad, Ram (1992). Tribal migration in Himalaya frontiers: Study of Gujjar Bakarwal Transhumance Economy Vintage Book, ISBN 81-85326-46-0.

Rao, P., Barik, S.K., Pandey, H.N. and Tripthi, R.S. (1990). Community composition and tree population structure in a sub-tropicalcal broad leaved forest along a disturbance gradient. Vegetatio. 88; 151.162,

Rashid, A. and Sharma, A. (2012). Exploration of economically important fodder plants of district Rajouri, Jammu and Kashmir state. International Journal of Life Science and Pharma Research, 2: 144-148.

Rashid, A., Anand, V. K. and Serwar, J. (2008). Less known Wild Edible Plants used by the Gujjar Tribe of District Rajouri, Jammu and Kashmir State, India; Int. J.Bot.4 (2): 219- 224.

Schultes, R.E. (1962). The role of ehtnobotanists in search for new medicinal plants, Llyodia, 25: 257-261.

Sharma, B.M. and Kachroo, P. (1983). Flora of Jammu and Plants of neighborhood. Bishen Singh Mahendra Pal Singh, Dehra Dun.

Singh, A., Dangwal, L.R. and Singh, T. (2014). Grassy weeds of district Rajouri, Jammu \& Kashmir, India, Pak. J. Weed Sci. Res., 20(2): 265-277

Singh, K.K. \& Kumar, K. (2000). Ethnobotanical wisdom of Gaddi tribes in western Himalaya (Bishen Singh Mahendra Pal Singh, Dehra Dun).

Swami, A. and Gupta, B.K. (1998). Flora of Udhampur, Bishan Singh Mahendra Pal Singh, Dehra Dun.

Vetaas, O.R. (1993). Spatial and temporal vegetation changes along a moisture gradient in northeastern $\mathrm{Su}-$ dan. Biotropica, 25: 164-175. 